\title{
Levenberg-Marquardt Method Based Iterative Square Root Cubature Kalman Filter and its Applications to Maneuvering Re-entry Target Tracking
}

\author{
Mu Jing \\ School of Computer Science and Engineering \\ Xi'an Technological University \\ Xi'an, 710032, China \\ e-mail: mujing1977@163.com
}

\author{
Wang Changyuan \\ School of Computer Science and Engineering \\ Xi'an Technological University \\ Xi'an, 710032, China \\ e-mail: cyw901@163.com
}

\begin{abstract}
Levenberg-Marquardt (abbr.L-M) method based iterative square root cubature Kalman filter (abbr. ISRCKFLM) inherits the numerical stability of square root Cubature Kalman filter and effectively suppresses the influence of the larger initial estimation error and the nonlinearity of the measurement equation on the state estimation in the nonlinear state estimation due to obtaining the optimal state and variance estimates using the latest measurement through L-M method. We apply the ISRCKFLM algorithm to the state estimation of maneuvering re-entry target tracking, the simulation results demonstrate that the ISRCKFLM algorithm has better accuracy of state estimation, comparable to Unscented Kalman filter and square root Cubature Kalman filter, according to estimation error analysis of the position, velocity, drag coefficient, turn coefficient and climbing force coefficient, and has fast convergence rate.
\end{abstract}

Keywords-Nonlinear Filtering; Square Root Cubature Kalman Filter; Levenberg-Marquardt Method; Maneuvering Reentry Targets Tracking

\section{INTRODUCTION}

The state estimate of maneuver re-entry target is highly non-linear filtering problem and has been much attention in the academic and engineering domain $[1,2]$. Up to now, the commonly used non-linear filtering is the extended Kalman filter (EKF) [3]. The EKF is based on first-order Taylor approximations of state transition and observation equation about the estimated state trajectory under Gaussian assumption, so EKF may introduce significant bias, or even convergence problems due to the overly crude approximation [4]. Especially, for highly nonlinear problems such as state estimation of maneuver re-entry target, EKF may produce large filtering errors and even divergence. Based on the Unscented Transformation (UT), the Unscented Kalman filter (UKF) use a set of deterministic sampling points to approximate the posterior probability distribution of the system state, that is, the Sigma points are used to capture the mean and variance information of the state [5].

Recently, as a new way to solve the nonlinear estimation problem, cubature rules based cubature Kalman filter (CKF) proposed in [6] uses numerical multi-dimensional integral to approximate the recursive Bayesian estimation integrals under the Gaussian assumption. The CKF can solve high- dimensional nonlinear filtering problems with minimal computational effort. Then the square root cubature Kalman filter algorithm (SRCKF) was proposed in order to improve the numerical stability [6].

On the other hand, in order to decrease the effect of initial estimation error and nonlinearity of measurement equation, Levenberg-Marquardt method based iterative square root cubature Kalman filter (ISRCKFLM) was developed on the basis of the SRCKF in [7]. In this paper, we apply the ISRCKFLM algorithm to state estimation of maneuver re-entry target. Simulations demonstrate that the ISRCKFLM algorithm can greatly improve the tracking accuracy of maneuver re-entry target and obtain fast convergence, compared with the UKF and SRCKF algorithms.

The rest of the paper is organized as follows. We begin with a description of the ISRCKFLM algorithm in Section 2. Then we apply the ISRCKFLM algorithm to track re-entry ballistic target (RBT) with unknown ballistic coefficient and discuss the simulation results in Section 3. Finally, we draw conclusion in Section 4.

\section{L-M BASED ITERATIVE SQUARE ROOT CUBATURE KALMAN FILTER}

Consider the following non-linear dynamics system:

$$
\begin{gathered}
\boldsymbol{x}_{k}=\boldsymbol{f}\left(\boldsymbol{x}_{k-1}\right)+\boldsymbol{w}_{k-1} . \\
\boldsymbol{z}_{k}=\boldsymbol{h}\left(\boldsymbol{x}_{k}\right)+\boldsymbol{v}_{k} .
\end{gathered}
$$

where $\boldsymbol{f}$ and $\boldsymbol{h}$ are some known nonlinear functions; $\boldsymbol{x}_{k} \in \mathbb{R}^{n_{x}}$ and $\boldsymbol{z}_{k} \in \mathbb{R}^{n_{z}}$ is state and the measurement vector, respectively; $\boldsymbol{w}_{k-1}$ and $\boldsymbol{v}_{k}$ are process and measurement Gaussian noise sequences with zero means and covariance $\boldsymbol{Q}_{k-1}$ and $\boldsymbol{R}_{k}$, respectively, and $\left\{\boldsymbol{w}_{k-1}\right\}$ and $\left\{\boldsymbol{v}_{k}\right\}$ are mutually uncorrelated.

Suppose that the state distribution at $k$-1 time is $\mathbf{x}_{k-1} \sim \mathcal{N}\left(\hat{\mathbf{x}}_{k-1}, \mathbf{S}_{k-1} \mathbf{S}_{k-1}^{T}\right)$, Levenberg-Marquardt based Iterative square root cubature Kalman filter (ISRCKFLM) is described as follows. 


\section{A. Time Update}

1) Calculate the cubature points and propagate the cubature points through the state equation

$$
\begin{gathered}
\boldsymbol{X}_{i, k-1}=\boldsymbol{S}_{k-1} \boldsymbol{\xi}_{i}+\hat{\boldsymbol{x}}_{k-1} . \\
\boldsymbol{X}_{i, k}^{*}=\boldsymbol{f}\left(\boldsymbol{X}_{i, k-1}\right) .
\end{gathered}
$$

where $\boldsymbol{\xi}_{i}=\sqrt{m / 2}[1]_{i}, \omega_{i}=1 / m, i=1, \cdots m=2 n_{x}$, the $[1]_{i}$ is a $n_{x}$ dimensional vector and is generated according to the way described in [2].

2) Evaluate the predicted state and square root of the predicted covariance

$$
\begin{gathered}
\overline{\boldsymbol{x}}_{k}=\sum_{i=1}^{m} \omega_{i} \boldsymbol{X}_{i, k}^{*} . \\
\overline{\boldsymbol{S}}_{k}=\operatorname{Tria}\left(\left[\chi_{k}^{*} \boldsymbol{S}_{Q, k-1}\right]\right) .
\end{gathered}
$$

here, $\boldsymbol{S}_{Q, k-1}$ denotes a square-root factor of $\boldsymbol{Q}_{k-1}$ and Tria() is denoted as a general triagularization algorithm. The matrix $\chi_{k}^{*}$ is defined as:

$$
\chi_{k}^{*}=1 / \sqrt{m}\left[\boldsymbol{X}_{1, k}^{*}-\overline{\boldsymbol{x}}_{k} \boldsymbol{X}_{2, k}^{*}-\overline{\boldsymbol{x}}_{k}, \cdots, \boldsymbol{X}_{m, k}^{*}-\overline{\boldsymbol{x}}_{k}\right] .
$$

3) Evaluate the modified covariance:

$$
\tilde{\boldsymbol{P}}_{k}=\left[\boldsymbol{I}-\overline{\boldsymbol{S}}_{k} \overline{\boldsymbol{S}}_{k}^{T}\left(\overline{\boldsymbol{S}}_{k} \overline{\boldsymbol{S}}_{k}^{T}+\frac{1}{\mu_{i}} \boldsymbol{I}\right)^{-1}\right] \overline{\boldsymbol{S}}_{k} \overline{\boldsymbol{S}}_{k}^{T} .
$$

where $\mu_{i}$ is adjusting parameter.

\section{B. Measurement update}

1) Set the initial value as: $\hat{\boldsymbol{x}}_{k}^{(0)}=\overline{\boldsymbol{x}}_{k}$.

2) Assuming the $i$-th iterate ${ }_{k}^{(i)}$, calculate the matrix

$$
\boldsymbol{L}_{k}^{(i)}=\tilde{\boldsymbol{P}}_{k} \boldsymbol{J}_{h}^{T}\left(\hat{\boldsymbol{x}}_{k}^{(i)}\right)\left[\boldsymbol{J}_{h}\left(\hat{\boldsymbol{x}}_{k}^{(i)}\right) \tilde{\boldsymbol{P}}_{k} \boldsymbol{J}_{h}^{T}\left(\hat{\boldsymbol{x}}_{k}^{(i)}\right)+\boldsymbol{R}_{k}\right]^{-1} .
$$

3) Calculate the i-th iterate

$$
\begin{aligned}
\hat{\boldsymbol{x}}_{k}^{(i+1)}= & \overline{\boldsymbol{x}}_{k}+\boldsymbol{L}_{k}^{(i)} \\
& \left\{\boldsymbol{z}_{k}-\boldsymbol{h}\left(\boldsymbol{x}_{k}^{(i)}\right)-\boldsymbol{J}_{h}\left(\hat{\boldsymbol{x}}_{k}^{(i)}\right)\left(\overline{\boldsymbol{x}}_{k}-\hat{\boldsymbol{x}}_{k}^{(i)}\right)\right\} . \\
& -\mu_{i}\left\{\boldsymbol{I}-\boldsymbol{L}_{k}^{(i)} \boldsymbol{J}_{h}\left(\hat{\boldsymbol{x}}_{k}^{(i)}\right)\right\} \tilde{\boldsymbol{P}}_{k}\left(\overline{\boldsymbol{x}}_{k}-\hat{\boldsymbol{x}}_{k}^{(i)}\right)
\end{aligned}
$$

4) Calculate the iteration termination condition

$$
\left\|\hat{\boldsymbol{x}}_{k}^{(i+1)}-\hat{\boldsymbol{x}}_{k}^{(i)}\right\| \leq \varepsilon \text { or } i=N_{\max } .
$$

$\varepsilon$ and $N_{\text {max }}$ are predetermined threshold and maximum iterate number, respectively. If the termination condition meets, the iterate return to 5); otherwise, set $\hat{\boldsymbol{x}}_{k}^{(i)}=\hat{\boldsymbol{x}}_{k}^{(i+1)}$, continue to 2).

5) Calculate the state estimation at time instant

$$
\hat{\boldsymbol{x}}_{k}=\hat{\boldsymbol{x}}_{k}^{(N)} .
$$

6) Evaluate the cross-covariance and square root of innovation covariance at $k$ time

$$
\begin{gathered}
\boldsymbol{P}_{x z}=\overline{\boldsymbol{S}}_{k} \overline{\boldsymbol{S}}_{k}^{T} \boldsymbol{J}_{h}^{T}\left(\hat{\boldsymbol{x}}_{k}^{(N)}\right) . \\
\boldsymbol{S}_{z z}=\operatorname{Chol}\left(\left[\boldsymbol{J}_{h}\left(\hat{\boldsymbol{x}}_{k}^{(N)}\right) \overline{\boldsymbol{S}}_{k} \quad \boldsymbol{S}_{R, k}\right]\right) .
\end{gathered}
$$

7) Calculate the square root of covariance at k time

$$
\begin{aligned}
& \boldsymbol{K}_{k}=\boldsymbol{P}_{x z} / \boldsymbol{S}_{z z}^{T} / \boldsymbol{S}_{z z} . \\
& \boldsymbol{S}_{k}=\operatorname{Chol}\left(\left[\overline{\boldsymbol{S}}_{k}-\boldsymbol{K}_{k} \boldsymbol{J}_{h}\left(\hat{\boldsymbol{x}}_{k}^{(N)}\right) \overline{\boldsymbol{S}}_{k} \quad \boldsymbol{K}_{k} \boldsymbol{S}_{R, k}\right]\right) .
\end{aligned}
$$

where symbol "/" represents the matrix right divide operator.

\section{APPLICATIONS TO MANEUVERING REENTRY TARGET TRACKING}

In the simulation, the trajectory of the maneuvering reentry generated in [8] is used with the same parameters, initial state and covariance estimation. To compare the performance of the ISRCKFLM with the UKF and SRCKF algorithms, we obtain the root mean square errors (RMSEs) in the position, velocity, drag coefficient, turn coefficient and climb coefficient showed in the Figure 1-5. All performance curves were obtained by averaging over 100 independent Monte Carlo runs. Table.1 lists the accumulated root mean square errors (ARMSEs).

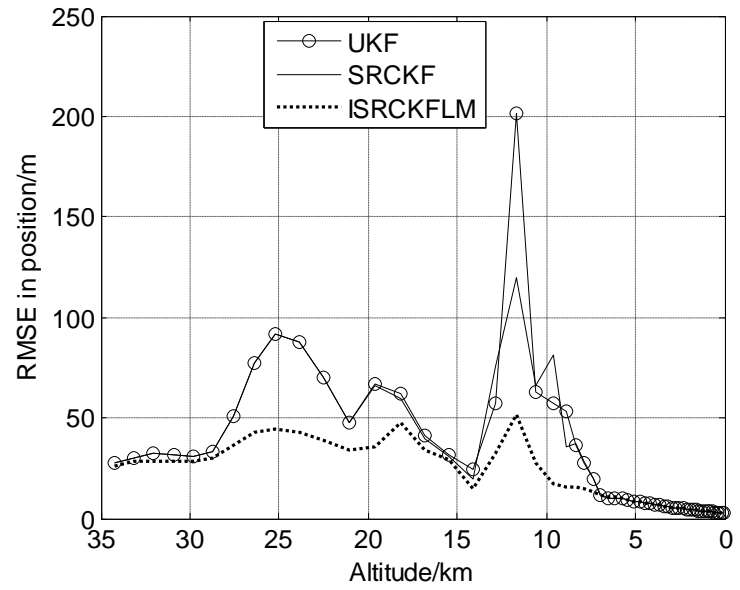

Figure 1. RMSE in Position

From Figur.1-2, we can see the ISRCKFLM's RMSEs in positon and velocity are lower than those of UKF and SRCKF algorithms, especially, the RMSEs of ISRCKFLM 
algorithm are effectively suppressed, and those of UKF and SRCKF algorithms have a big jump when the target experiences the high maneuver at altitude $23 \mathrm{~km}$ and $10 \mathrm{~km}$.

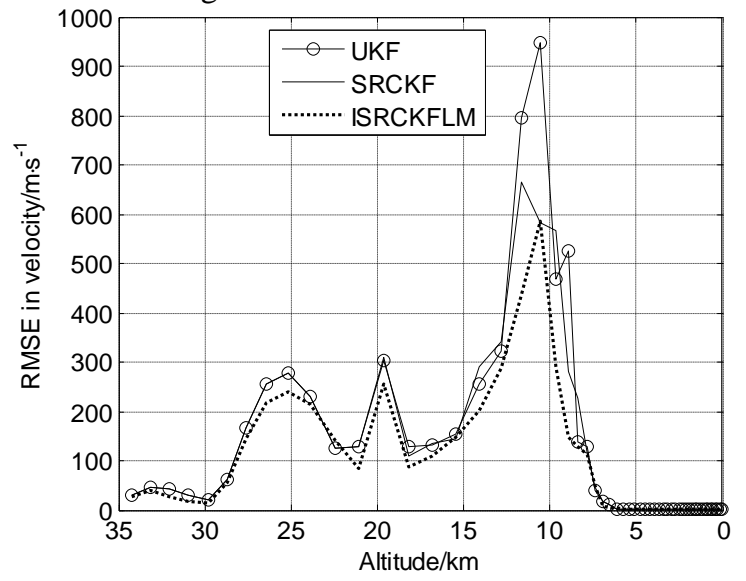

Figure 2. RMSE in Velocity

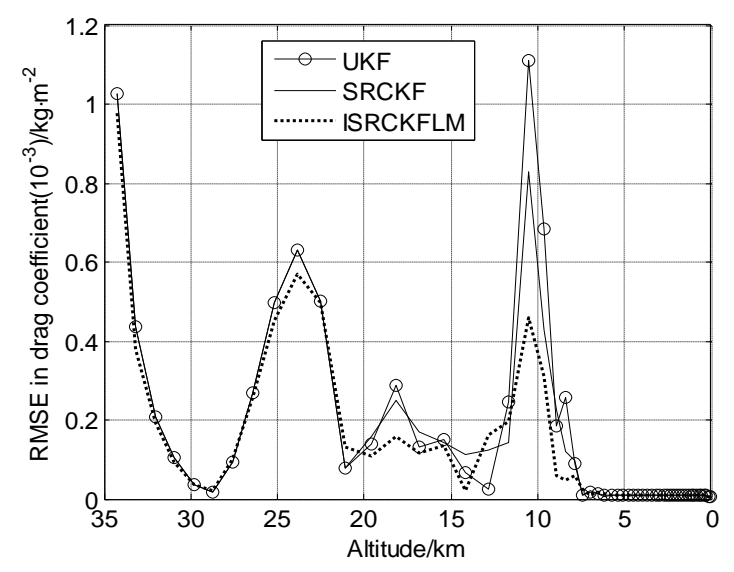

Figure 3. RMSE in Drag Coefficient

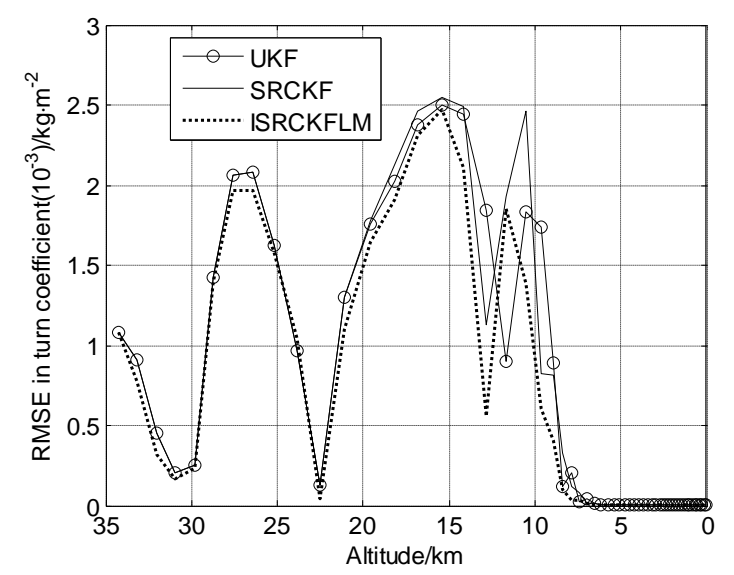

Figure 4. RMSE in Turn Coefficient

As for the estimate on drag coefficient, turn coefficient and climbing coefficient of maneuver re-entry target, from Figure.3-5we can see the RMSEs of three algorithms decrease between $35 \mathrm{~km}-30 \mathrm{~km}$ at altitude, and the RMSEs of three algorithms reach the smallest at about $30 \mathrm{~km}$ at altitude.
Then the RMSEs of three algorithms increases when a dive and turn maneuver occurs on re-entry target, however, the ISRCKFLM's RMSEs are lower than those of the other two algorithms. The RMSEs of all filters gradually increase when the target experiences an increased climb force at altitude $16 \mathrm{~km}-10 \mathrm{~km}$, but the ISRCKFLM's RMSE increases slightly slower. After the maneuver was withdrawn, the RMSE of the three algorithms began to decline.

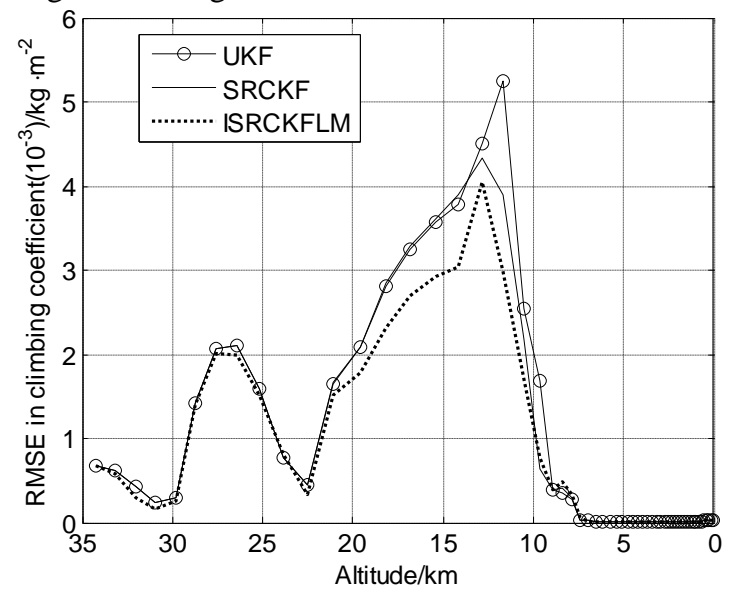

Figure 5. RMSE in Climbing Coefficient

TABLE I. ARMSES IN THREE FILTERS

\begin{tabular}{|c|c|c|c|c|c|}
\hline $\begin{array}{l}\text { Algori- } \\
\text { thms }\end{array}$ & $\begin{array}{c}A R M S E_{p} \\
/ \boldsymbol{m}\end{array}$ & $\begin{array}{c}A R M S E_{v} \\
/ \mathbf{m} \cdot \mathbf{s}^{-1}\end{array}$ & $\begin{array}{c}A R M S E_{d} / \\
\mathbf{k g} \cdot \mathbf{m}^{-2}\end{array}$ & $\begin{array}{c}A R M S E_{t} \\
/ \mathbf{k g} \cdot \mathbf{m}^{-2}\end{array}$ & $\begin{array}{c}A R M S E_{c} \\
/ \mathbf{k g} \cdot \mathbf{m}^{-2}\end{array}$ \\
\hline UKF & 42.3763 & 202.0984 & $\begin{array}{c}0.000277 \\
31\end{array}$ & $\begin{array}{c}0.001000 \\
4\end{array}$ & $\begin{array}{c}0.001468 \\
8\end{array}$ \\
\hline SRCKF & 38.9467 & 184.3961 & $\begin{array}{c}0.000251 \\
65\end{array}$ & $\begin{array}{c}0.000982 \\
07\end{array}$ & $\begin{array}{c}0.001386 \\
5\end{array}$ \\
\hline $\begin{array}{c}\text { ISRCKF } \\
\text { LM }\end{array}$ & 22.4556 & 141.8943 & $\begin{array}{c}0.000218 \\
02\end{array}$ & $\begin{array}{c}0.000899 \\
5\end{array}$ & $\begin{array}{c}0.001186 \\
1\end{array}$ \\
\hline
\end{tabular}

Moreover, the ARMSEs of the ISRCKFLM in the positon, velocity, drag coefficient, turn coefficient and climbing coefficient are lower than those of UKF and SRCKF algorithms from Table.1.

Therefore, on the basis of the simulation results presented in Figure.1-5, we can draw a conclusion that the ISRCKFLM yields on the superior performance over the UKF and SRCKF on the state estimation of maneuvering re-entry target.

\section{CONCLUSION}

In this paper we apply the ISRCKFLM algorithm to maneuvering re-entry target tracking. The latest measurement are fully used in the ISRCKFLM algorithm, and innovation covariance and cross-covariance are improved in the iterative process, so we can obtain the optimal state estimation and covariance estimation. Simulation results demonstrate that the performance of ISRCKFLM algorithm is superior to UKF and CKF algorithms by analysis of errors in position, velocity, drag 
coefficient, turn coefficient and climbing coefficient, and has the faster convergence rate.

\section{ACKNOWLEDGMENT}

The authors would like to thank the support of fund of the State and Local Joint Laboratory of Advanced Network and Monitoring Control Engineering (No.GSYSJ201603); Natural Science Basic Research Program of Department of science and technology of Shaanxi Province - Youth Foundation (No.2017JQ6056); The Key Project of Department of Education of Shaanxi Province (No.16JF012).

\section{REFERENCES}

[1] Li XR, Jilkov VP. A survey of maneuvering target tracking - part II: ballistic target models. Signal and Data Processing of Small Targets, 2001, 4473:559-581
[2] Farina A, Ristic B, Benvenuti D. Tracking a ballistic target: comparison of several nonlinear filters. IEEE Transactions on Aerospace and Electronic Systems, 2002, 38 (3):854-867.

[3] Bar-Shalom Y, Li XR, Kirubarajan T. Estimation with Applications to Tracking and Navigation. New York: John Wiley \&Son, Inc, 2001.

[4] Athans M, Wishner RP, Bertolini A. Suboptimal state estimation for continuous-time nonlinear system from discrete noisy measurements[J]. IEEE Transactions on Automatic Control, 1968, 13(5):504-514.

[5] Julier SJ, Uhlmann JK. Unscented filtering and nonlinear estimation. Proceedings of the IEEE, 2004, 92 (12):1958-1958.

[6] Arasaratnam I, Haykin S. Cubature Kalman filters. IEEE Transactions on Automatic Control, 2009, 54(6): 1254-1269.

[7] Mu. J.; Cai. Y.; Wang. C.Y. L-M Method Based Iteration Cubature Kalman Filter and its Applications, Journal of Xi'an Technological University, 2013, 33(1):1-6.

[8] Jing Mu, Yuanli Cai, Changyuan Wang. State estimation of Maneuvering Reentry Target Using Likelihood Based Iterated Divided Difference Filter, Journal of Xi' an Technological University, 32(5),349-354, 2012.5. 